\title{
Analysis of structurally discontinuous reinforced concrete building frames using the ASI technique
}

\author{
Daigoro Isobe ${ }^{\mathrm{a}, *}$, Yutaka Toi $^{\mathrm{b}}$ \\ ${ }^{a}$ Institute of Engineering Mechanics and Systems, University of Tsukuba, 1-1-1 Tennodai Tsukuba-shi, Ibaraki 305-8573, Japan \\ ${ }^{\mathrm{b}}$ Institute of Industrial Science, University of Tokyo, Tokyo, Japan
}

Received 11 December 1997; accepted 10 June 1999

\begin{abstract}
The Adaptively Shifted Integration (ASI) technique, which produces the highest computational efficiency in the finite element analyses of framed structures including static and dynamic collapse problems, is applied to structurally discontinuous problems of reinforced concrete building frames. A new numerical scheme based on the updated Lagrangian formulation (ULF) adaptation of the ASI technique is developed, by modeling the fracture of a section by a plastic hinge located at the exact position with a simultaneous release of resultant forces in the element. By using the algorithms described in this paper, the analyses became possible even by the conventional displacement-based finite element codes, and sufficiently reliable solutions for practical use have been obtained in the explosive demolition and seismic damage analyses of a five storied, five span RC building frame. The present technique can be easily implemented with minimum effort into the existing finite element codes utilizing the linear Timoshenko beam element. (C) 2000 Elsevier Science Ltd. All rights reserved.
\end{abstract}

Keywords: Adaptively Shifted Integration technique; Explosive demolition analysis; Seismic damage analysis; Member fracture; Timoshenko beam element; Reinforced concrete building; Finite element method

\section{Introduction}

The technology used in the demolition of old wornout buildings has always been of major interest and challenge in civil engineering. To meet the heavy demand for demolition work, a controlled explosion technique using high explosives has been developed in recent years as conventional demolition techniques using a hydraulic concrete crusher, a concrete cutter or a non-explosive demolition agent are lengthy and costly. In Japan, the explosive demolition technique has recently been used in a few cases, such as the demolition of international exposition buildings in Tsu-

\footnotetext{
* Corresponding author.
}

kuba and an unused hotel in Kyoto. The explosive demolition technique increases work efficiency but poses a high risk of damaging neighboring buildings, especially in urban areas. Therefore, a test of the assumptions using computational analysis made in devising the controlled explosive demolition, has become essential to ensure the success of this method.

The Great Hanshin-Awaji Earthquake, which occurred in January 1995, caused severe damage over a large area. As a result of this earthquake the structural design guidelines for buildings, especially against vertical seismic waves, were thoroughly reviewed and due consideration was given to the development of a convenient technique to analyze collapse modes of structural members under a three-directional excitation. 
So far, there are few numerical examples of computational schemes, such as the Distinct Element Method (DEM) [1] or the Discontinuous Deformation Analysis (DDA) [2], applied to demolition or seismic damage analyses [3-8]. The commonly used finite element codes can only be used after making complicated modifications to simulate dynamic collapse problems which contain strong nonlinearities and discontinuities, such as fractures occurring in flexural damage or shear damage in reinforced concrete members. The main purpose of this paper, is to devise a new algorithm applicable to these types of discontinuous problems by using the finite element method.

As shown in previous papers [9-12], the highest computational efficiency in finite element analyses of framed structures including the geometrically nonlinear, elasto-plastic analysis as well as the geometrically linear, plastic collapse analysis and the dynamic collapse analysis, has been achieved by using the Adaptively Shifted Integration (ASI) technique. In this paper, the ASI technique and the linear Timoshenko beam element, which can be easily implemented with a minimum effort into existing finite element codes, are extended and applied to problems of structural discontinuities in explosive demolition analysis and seismic damage analysis.

In this technique the numerical integration points in an elastically deformed beam element are placed at the optimal points for linear analysis (midpoint in the linear Timoshenko beam and Gaussian integration points in the cubic beam element) and are immediately shifted after the occurrence of a fully plastic section in the element, using previously established relations between the locations of numerical integration points and those of plastic hinges [13], to form a plastic hinge exactly at the position of the fully plastic section. In this manner this technique produces a higher computational accuracy with fewer elements than conventional finite element methods. An explosion or a fracture is represented in this paper by releasing the resultant forces in an element immediately after a plastic hinge is located at the section. Only the resultant forces are assumed as the release forces reacting on the element and thus, there are no impact phenomena assumed in the demolition process. With the proposed technique problems associated with modeling structural discontinuities, such as those mentioned above, can be easily tackled even by conventional displacement-based finite element codes. More details about this technique are given in subsequent sections of this paper.

Typically, the total Lagrangian formulation (hereafter abbreviated as TLF) and the updated Lagrangian formulation (ULF) [14] are used in incremental nonlinear structural analyses. As the occurrence of extremely large rotations and strains is anticipated in dynamic collapse analyses, the ULF is used in this paper. In order to maintain high computational efficiency, an explicit time integration scheme using the central difference method is chosen for explosive demolition analysis, so as to model the high frequency response better. On the other hand, the implicit time integration scheme using Newmark's $\beta$ method is chosen for seismic damage analysis, as this scheme is better suited for modeling low frequency response. Also, a distributed mass matrix is used in the implicit code to reduce response errors due to a rough finite element subdivision.

In Section 2, the explicit nonlinear code and the implicit nonlinear code based on the ULF are explained. In Section 3, the ASI algorithm for dynamic collapse analyses is described. The results of applying this model to explosive demolition analysis and seismic damage analysis of a five storied, five span reinforced concrete building frame are detailed in Section 4. Section 5 contains concluding remarks.

\section{Time integration schemes based on the ULF}

In this section, an explicit time integration scheme based on the ULF applied in explosion demolition analyses and an implicit time integration scheme based on the ULF applied in seismic damage analyses, are described.

\subsection{Relations between generalized strain increments and nodal displacement increments}

The relations between the generalized strain increments and the nodal displacement increments of a linear Timoshenko beam element at incremental step $n$, are expressed as

$$
\begin{aligned}
& \left\{\begin{array}{l}
\Delta_{n} \bar{\varepsilon}_{1} \\
\Delta_{n} \bar{\varepsilon}_{2} \\
\Delta_{n} \bar{\varepsilon}_{3} \\
\Delta_{n} \bar{\varepsilon}_{4} \\
\Delta_{n} \bar{\varepsilon}_{5} \\
\Delta_{n} \bar{\varepsilon}_{6}
\end{array}\right\}= \\
& \left\{\begin{array}{l}
\left(\Delta_{n} \bar{\theta}_{x J}-\Delta_{n} \bar{\theta}_{x I}\right) /{ }^{n} l \\
\left(\Delta_{n} \bar{\theta}_{y J}-\Delta_{n} \bar{\theta}_{y I}\right) /{ }^{n} l \\
\left(\Delta_{n} \bar{w}_{J}\right)-\Delta_{n} \bar{w}_{I} /{ }^{n} l \\
\left(\Delta_{n} \bar{\theta}_{z J}-\Delta_{n} \bar{\theta}_{z I}\right) /{ }^{n} l \\
\left(\Delta_{n} \bar{u}_{J}-\Delta_{n} \bar{u}_{I}\right) /{ }^{n} l-\Delta_{n} \bar{\theta}_{y I} .(1-s) / 2-\Delta_{n} \bar{\theta}_{y J} .(1+s) / 2 \\
\left(\Delta_{n} \bar{v}_{J}-\Delta_{n} \bar{v}_{I}\right) /{ }^{n} l+\Delta_{n} \bar{\theta}_{x I} .(1-s) / 2+\Delta_{n} \bar{\theta}_{x J} .(1+s) / 2
\end{array}\right\}
\end{aligned}
$$


Here, it should be noted that the nodal displacement increments are based on the elemental coordinate at incremental step $n$. The element length ${ }^{n} l$ calculated at incremental step $n$ is used to evaluate the generalized strain increments, and the strain increments are equal to the updated Green strain increments.

The generalized strain increment vector and the nodal displacement increment vector are expressed as

$$
\left\{\Delta_{n} \bar{\varepsilon}\right\}=\left[{ }_{n}^{n} \bar{B}_{L}\right] \cdot\left\{\Delta_{n} \bar{u}\right\}
$$

$$
\left\{\Delta_{n} \bar{u}\right\}=\left[{ }^{u} T\right] \cdot\left[{ }^{0} T\right] \cdot\{\Delta u\}
$$

where

$$
\begin{gathered}
\{\Delta u\}^{\mathrm{T}}=\left[\Delta u_{I}, \Delta v_{I}, \Delta w_{I}, \Delta \theta_{x I}, \Delta \theta_{y I}, \Delta \theta_{z I}, \Delta u_{J}, \Delta v_{J},\right. \\
\left.\Delta w_{J}, \Delta \theta_{x J}, \Delta \theta_{y J}, \Delta \theta_{z J}\right]
\end{gathered}
$$

$\left[{ }^{0} T\right]$ and $\left[{ }^{u} T\right]$ in Eq. (3) are the transformation matrix from global coordinates to the initial elemental coordinates, and the transformation matrix from the initial elemental coordinates to elemental coordinates at step $n$, respectively. In this study $\left[{ }^{u} T\right]$ is calculated by successive iteration and is expressed as follows:

$$
\left[{ }^{u} T\right]=\left[{ }^{n} T\right] \cdot\left[{ }^{n-1} T\right] \cdot\left[{ }^{n-2} T\right] \ldots\left[\left[{ }^{3} T\right] \cdot\left[{ }^{2} T\right] \cdot\left[{ }^{1} T\right]\right.
$$

where $\left[{ }^{n} T\right]$ is the transformation matrix from elemental coordinates at step $(n-1)$ to elemental coordinates at step $n$. The matrix $\left[{ }^{n} T\right]$ is calculated as follows:

$\left[{ }^{n} T\right]=\left[\begin{array}{llll}{ }^{n} T^{*} & 0 & 0 & 0 \\ 0 & { }^{n} T^{*} & 0 & 0 \\ 0 & 0 & { }^{n} T^{*} & 0 \\ 0 & 0 & 0 & { }^{n} T^{*}\end{array}\right]$

where

$$
\begin{aligned}
& {\left[{ }^{n} T^{*}\right]=\left[{ }^{n} T^{\gamma}\right] \cdot\left[{ }^{n} T^{\beta}\right] \cdot\left[{ }^{n} T^{\alpha}\right]} \\
& =\left[\begin{array}{lll}
\cos \left({ }^{n} \gamma\right) & \sin \left({ }^{n} \gamma\right) & 0 \\
-\sin \left({ }^{n} \gamma\right) & \cos \left({ }^{n} \gamma\right) & 0 \\
0 & 0 & 1
\end{array}\right] \\
& \times\left[\begin{array}{lll}
\cos \left({ }^{n} \beta\right) & 0 & -\sin \left({ }^{n} \beta\right) \\
0 & 1 & 0 \\
\sin \left({ }^{n} \beta\right) & 0 & \cos \left({ }^{n} \beta\right)
\end{array}\right] \\
& \times\left[\begin{array}{lll}
1 & 0 & 0 \\
0 & \cos \left({ }^{n} \alpha\right) & -\sin \left({ }^{n} \alpha\right) \\
0 & \sin \left({ }^{n} \alpha\right) & \cos \left({ }^{n} \alpha\right)
\end{array}\right]
\end{aligned}
$$

By defining the nodal displacement increments between step $(n-1)$ and $n$ as $\Delta_{n-1} \bar{u}_{I}, \Delta_{n-1} v_{I}, \Delta_{n-1} w_{I}, \ldots \Delta_{n-1} \bar{\theta}_{x J}$,
$\Delta_{n-1} \bar{\theta}_{y J}$ and $\Delta_{n-1} \bar{\theta}_{z J}$ respectively, $\cos \left({ }^{n} \alpha\right), \cos \left({ }^{n} \beta\right)$ and ${ }^{n} \gamma$ in Eq. (7) can be calculated as follows:

$\cos \left({ }^{n} \alpha\right)=$

$\frac{\left\{{ }^{n-1} l+\left(\Delta_{n-1} \bar{w}_{J}-\Delta_{n-1} \bar{w}_{I}\right)\right\}}{\left[\left\{{ }^{n-1} l+\left(\Delta_{n-1} \bar{w}_{J}-\Delta_{n-1} \bar{w}_{I}\right)\right\}^{2}+\left(\Delta_{n-1} \bar{v}_{J}-\Delta_{n-1} \bar{u}_{I}\right)^{2}\right]^{1 / 2}}$

$$
\begin{aligned}
& \cos \left({ }^{n} \beta\right)= \\
& \frac{\left\{{ }^{n-1} l+\left(\Delta_{n-1} \bar{w}_{J}-\Delta_{n-1} \bar{w}_{I}\right)\right\}}{\left[\left\{{ }^{n-1} l+\left(\Delta_{n-1} \bar{w}_{J}-\Delta_{n-1} \bar{w}_{I}\right)\right\}^{2}+\left(\Delta_{n-1} \bar{u}_{J}-\Delta_{n-1} \bar{u}_{I}\right)^{2}\right]^{1 / 2}}
\end{aligned}
$$

${ }^{n} \gamma=\left(\Delta_{n-1} \bar{\theta}_{z I}+\Delta_{n-1} \bar{\theta}_{z J}\right) / 2$

where ${ }^{n-1} l$ is the element length at step $(n-1)$.

\subsection{Relations between resultant force increments and generalized strain increments}

The relation between the resultant force increment vector and the generalized strain increment vector of a linear Timoshenko beam element is expressed as

$$
\left\{\Delta_{n} \bar{R}\right\}=[D] \cdot\left\{\Delta_{n} \bar{\varepsilon}\right\}
$$

where $[D]$ is the stress-strain matrix. For an elastic element $[D]$ is given as

$$
[D]=\left[D^{e}\right]
$$

$$
=\left[\begin{array}{llllll}
E I_{x} & 0 & 0 & 0 & 0 & 0 \\
0 & E I_{y} & 0 & 0 & 0 & 0 \\
0 & 0 & E A & 0 & 0 & 0 \\
0 & 0 & 0 & G K & 0 & 0 \\
0 & 0 & 0 & 0 & \alpha_{x} G A & 0 \\
0 & 0 & 0 & 0 & 0 & \alpha_{y} G A
\end{array}\right]
$$

where $E, G, A, K, I_{x}, I_{y}, \alpha_{x}$ and $\alpha_{y}$ are Young's modulus, shear modulus, cross-sectional area, Saint-Venant's torsional coefficient, the moments of area inertia about $x$ and $y$ axes, and shear correction factors in the $x$ and $y$ axes, respectively. When the element becomes plastic the stress-strain matrix $[D]$ can be expressed as

$$
\begin{aligned}
{[D]=\left[D^{p}\right]=} & {\left[D^{e}\right] } \\
& -\frac{\left[D^{e}\right] \cdot\{\partial f / \partial \bar{R}\} \cdot\lfloor\partial f / \partial \bar{R}\rfloor \cdot\left[D^{e}\right]}{\lfloor\partial f / \partial \bar{R}\rfloor \cdot\left[D^{e}\right] \cdot\{\partial f / \partial \bar{R}\}}
\end{aligned}
$$

where $f$ is the plastic potential, which is given as 
$f=\left(\frac{\bar{R}_{1}}{M_{x 0}}\right)^{2}+\left(\frac{\bar{R}_{2}}{M_{y 0}}\right)^{2}+\left(\frac{\bar{R}_{3}}{N_{0}}\right)^{2}+\left(\frac{\bar{R}_{4}}{M_{z 0}}\right)^{2}-1$

where $\bar{R}_{1}, \bar{R}_{2}, \bar{R}_{3}$ and $\bar{R}_{4}$ are associated with the two components of bending moment, axial force and torsional moment, respectively. The effect of shear forces on the yield condition has been neglected in this study. The subscript ' 0 ' indicates a fully plastic value under the condition when each component of the resultant forces acts independently on the cross section of the member.

The strain vector and the stress vector at step $(n+$ 1) can be obtained by transforming the updated Kirchhoff stress increment vector to the Jaumann differential form vector, which is expressed as follows:

$\left\{{ }_{n}^{n+1} \bar{\varepsilon}\right\}=\left\{\begin{array}{l}n-\bar{\varepsilon} \\ n\end{array}\right\}+\left\{\Delta_{n} \bar{\varepsilon}\right\}$

$\left\{\Delta \bar{R}^{J}\right\}=\left[{ }^{n+1} A\right]\left\{\Delta_{n} \bar{R}\right\}$

$\left\{{ }_{n}^{n+1} \bar{R}\right\}=\left\{{ }_{n}^{n} \bar{R}\right\}+\left\{\Delta \bar{R}^{J}\right\}$

The transformation matrix $\left[{ }^{n+1} A\right]$ is expressed as

$\left.{ }^{n+1} A\right]=$

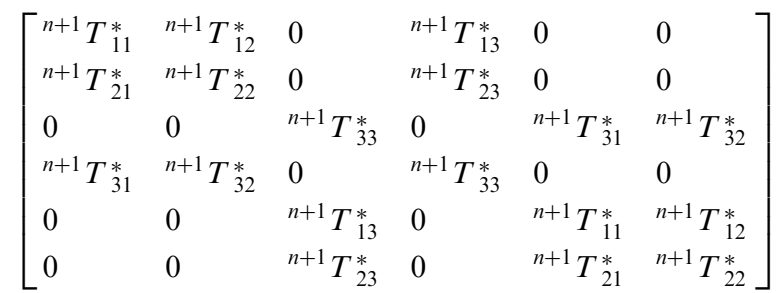

where ${ }^{n+1} T_{i j}^{*}$ is the $(i, j)$ term of the matrix $\left[{ }^{n+1} T^{*}\right]$ in Eq. (7)

\subsection{Explicit time integration scheme using central difference method}

Generally, an explicit time integration scheme is used when numerical models are controlled by a high frequency response. Therefore, the explicit code is chosen in explosive demolition analysis, in order to maintain high computational efficiency. In this section, the explicit code used in the analysis, is described.

The dynamic equilibrium equation at $t=t_{n}$ can be formulated as follows:

$[M]\left\{{ }^{n} \ddot{u}\right\}=\left\{{ }^{n} E\right\}-\left\{{ }_{n}^{n} F\right\}$

where $[M],\left\{{ }^{n} \ddot{u}\right\},\left\{{ }^{n} E\right\}$ and ${ }_{n}^{n} F$ are mass matrix, accel- eration vector at step $n$, nodal external force vector at step $n$ and internal force vector at step $n$, respectively.

The lumped mass matrix $[M]$ used in the explicit code is given by

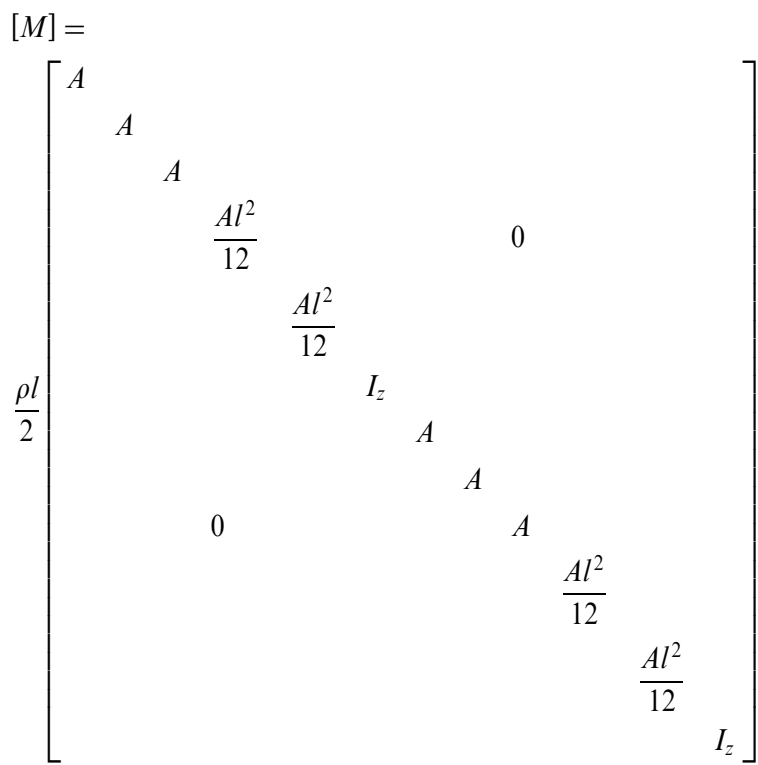

where $\rho, A, l$, and $I_{z}$ are density of the member, crosssectional area, length of the element and polar moment of area inertia, respectively.

In the present code, the acceleration vector $\{\ddot{u}\}$ at $t-t_{n}$ is approximated by the following central difference equation:

$\left\{{ }^{n} \ddot{u}\right\}=\left(\left\{{ }^{n-1} u\right\}-2\left\{^{n} u\right\}+\left\{{ }^{n+1} u\right\}\right) /(\Delta t)^{2}$

The substitution of Eq. (21) into Eq. (19) leads to

$$
\begin{aligned}
{[M]\left\{{ }^{n+1} u\right\}=} & \Delta t^{2}\left(\left\{{ }^{n} E\right\}-\left\{{ }_{n}^{n} F\right\}\right)+[M]\left(2\left\{{ }^{n} u\right\}\right. \\
& \left.-\left\{{ }^{n-1} u\right\}\right)
\end{aligned}
$$

where

$\left\{{ }_{n}^{n} F\right\}=\int_{{ }_{n}}\left[{ }^{0} T\right]^{\mathrm{T}} \cdot\left[{ }^{u} T\right]^{\mathrm{T}} \cdot\left[{ }_{n}^{n} \bar{B}_{L}\right]^{\mathrm{T}} \cdot\left\{{ }_{n}^{n} \bar{R}\right\} \mathrm{d} l$

Using the above equations, the displacement vector $\left\{{ }^{n+1} u\right\}$ can be calculated when the vectors $\left\{{ }^{n} u\right\}$ and $\left\{{ }^{n-1} u\right\}$ are known. However, in case of $n=0$ in Eq. (22), the vectors $\left\{{ }^{0} u\right\}$ and $\left\{^{-1} u\right\}$ are required. The vector $\left\{{ }^{0} u\right\}$ is given by the initial condition and the vector $\left\{{ }^{-1} u\right\}$ can be calculated by the following equation:

$$
\left\{{ }^{-1} u\right\}=\left\{{ }^{0} u\right\}-\Delta t\left\{{ }^{0} \dot{u}\right\}+\frac{(\Delta t)^{2}}{2}\left\{{ }^{0} \ddot{u}\right\}
$$


where the vector $\left\{{ }^{0} \dot{u}\right\}$ is the initial condition and $\left\{^{0} \ddot{u}\right\}$ is calculated by placing $t=0(n=0)$ in Eq. (19).

It is known that the time increment $\Delta t$ for the explicit scheme has to satisfy the following condition, known as Courant's stability condition:

$\Delta t<\Delta t_{\mathrm{cr}}=\frac{l_{\min }}{v_{\mathrm{L}}}$

where $v_{\mathrm{L}}$ is longitudinal wave velocity in an elastic solid, and $l_{\min }$ is length of the shortest element in the structure. Although there is the possibility of an increase in total computing time, the calculation time at each incremental step is reduced, as the present code requires no inverse matrices and attended processes. This also leads to a reduced demand for computer memory.

\subsection{Implicit time integration scheme using Newmark's $\beta$ method}

As an explicit code uses a lumped mass matrix which is simplified by lumping each element's mass at each node of an element, the numerical error tends to increase with time, in response analyses. Accordingly, in the ASI technique, which requires less amount of element subdivision, the numerical error caused by the use of lumped mass matrices is increased and becomes apparent. Thus, a distributed mass matrix should be used when numerical models are controlled by low frequency response, in cases such as seismic damage analysis. In this section, an implicit code implemented with a distributed mass matrix, is explained.

The following equation is substituted into Eq. (19) at $t=t_{n+1}$ in the implicit code:

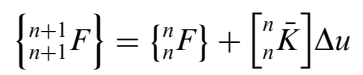

and the following incremental stiffness equation is evaluated:

$[M]\left\{{ }^{n+1} \ddot{u}\right\}+\left[{ }_{n}^{n} \bar{K}\right]\{\Delta u\}=\left\{{ }^{n+1} E\right\}-\left\{{ }_{n}^{n} F\right\}$

By neglecting residual forces, an implicit code is obtained by evaluating the following incremental kinematic equation:

$[M]\{\Delta \ddot{u}\}+\left[{ }_{n}^{n} \bar{K}\right]\{\Delta u\}=0$

where $\left[{ }_{n}^{n} \bar{K}\right]$ is a stiffness matrix at step $n$. The distributed mass matrix $[M]$ used in the implicit code is given by

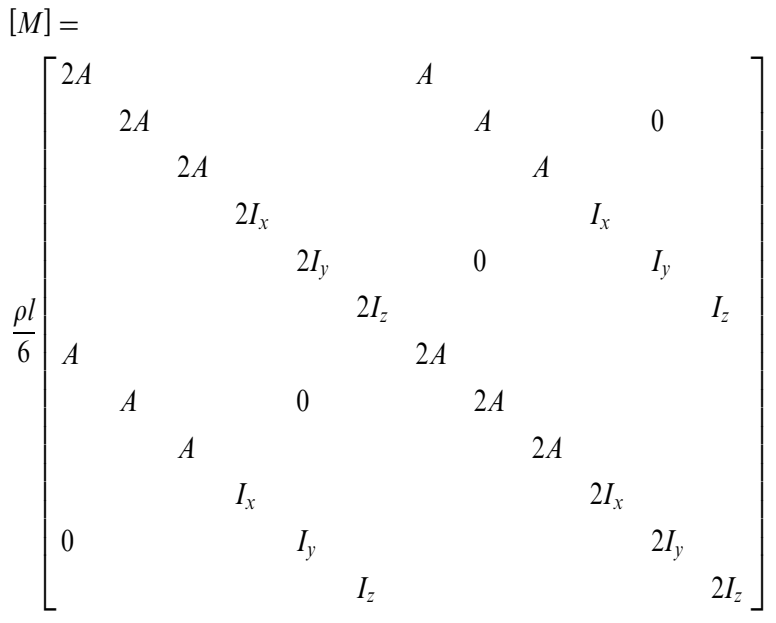

where $\rho, A, l, I_{x}, I_{y}$ and $I_{z}$ are density of the member, cross-sectional area, length of element, moments of area inertia about $\mathrm{x}$ and $\mathrm{y}$ axes and polar moment of area inertia, respectively.

In this paper the incremental kinematic equation for a structure under excitation at fixed points is as follows:

$$
\begin{aligned}
& {\left[M_{1}\right]\{\Delta \ddot{u}\}+\left[M_{2}\right]\left\{\Delta \ddot{u}_{b}\right\}+\left[{ }_{n}^{n} \bar{K}_{1}\right]\{\Delta u\}+\left[{ }_{n}^{n} \bar{K}_{2}\right]} \\
& \quad \times\left\{\Delta u_{b}\right\} \\
& =0
\end{aligned}
$$

The subscript ' 1 ' indicates the coupled terms between free nodal points, ' 2 ' indicates the coupled terms between free nodal and fixed nodal points, and ' $b$ ' indicates the components at fixed nodal points. Vectors $\{\Delta \ddot{u}\}$ and $\{\Delta u\}$ are the nodal acceleration increments and the nodal displacement increments, respectively.

On the assumption that the displacements at free nodal points are estimated by adding quasi-static displacement increments $\left\{\Delta u_{\mathrm{s}}\right\}$ and dynamic displacement increments $\left\{\Delta u_{d}\right\}$, the displacements at free nodal points are given as:

$\{\Delta u\}=\left\{\Delta u_{\mathrm{s}}\right\}+\left\{\Delta u_{\mathrm{d}}\right\}$

$\left\{\Delta u_{\mathrm{s}}\right\}$ is evaluated by neglecting inertia force as follows:

$\left\{\Delta u_{\mathrm{s}}\right\}=-\left[{ }_{n}^{n} \bar{K}_{1}\right]^{-1}\left[{ }_{n}^{n} \bar{K}_{2}\right]\left\{\Delta \ddot{u}_{b}\right\}$

Substituting Eqs. (31) and (32) into Eq. (30), the following equation is obtained: 


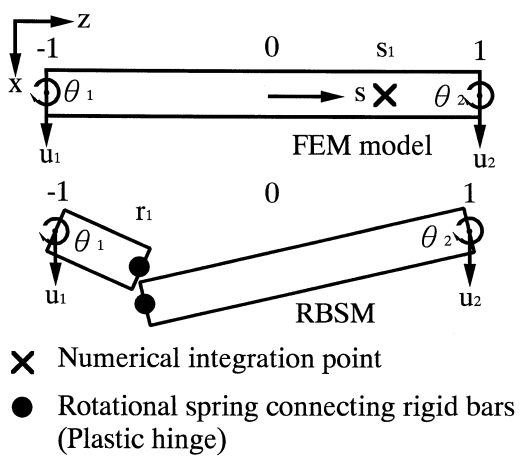

Fig. 1. Linear Timoshenko beam element and its physical equivalent.

$$
\begin{aligned}
& {\left[M_{1}\right]\left\{\Delta \ddot{u}_{\mathrm{d}}\right\}+\left[{ }_{n}^{n} \bar{K}_{1}\right]\left\{\Delta u_{\mathrm{d}}\right\}} \\
& \quad=\left(\left[M_{1}\right]\left[{ }_{n}^{n} \bar{K}_{1}\right]^{-1}\left[{ }_{n}^{n} \bar{K}_{2}\right]-\left[M_{2}\right]\right)\left\{\Delta \ddot{u}_{\mathrm{b}}\right\}
\end{aligned}
$$

In this scheme, equivalent forces are calculated by substituting nodal acceleration increments at fixed points into the right side of the above equation and the incremental kinematic equation is solved by Newmark's $\beta$ method.

Basically, it is possible to set the time increment in implicit codes longer than those in explicit codes. Although it is possible to reduce the total computational time by using a longer time increment, the evaluation of incremental equations at each step makes the calculation time per step longer, and, in addition, it leads to an excessive use of computer memory. Therefore, the conjugate gradient (CG) method is used as a solver in this study, to reduce the excessive demand on computer memory. Furthermore, damping matrices are not considered in this study, in order to evaluate the structural response on the conservative side.

\section{ASI technique for dynamic collapse analyses}

In this section, the algorithm for implementing the ASI technique as applied to dynamic collapse analysis, is explained. A relation between the location of a numerical integration point $\left(s_{1}\right)$ and occurrence of a plastic hinge $\left(r_{1}\right)$ in the linear Timoshenko beam element $\left(-1 \leq r_{1}, s_{1} \leq 1\right)$, is obtained [13] by considering the equivalence conditions between the strain energy approximations of a linear Timoshenko beam element and a physical model known as the rigid-bodies spring model (RBSM). Referring to Fig. 1, the relation is expressed by the following equation: $s_{1}=-r_{1} \quad$ or $\quad r_{1}=-s_{1}$

where $s_{1}$ and $r_{1}$ are positions of the numerical integration point and of the plastic hinge or member fracture, respectively.

When the entire region in an element behaves elastically, the midpoint of the element $\left(s_{1}=0\right)$ is the most appropriate integration point from considerations of accuracy and symmetry. The internal force vector at step $n$, based on the ULF, is expressed as

$\left\{{ }_{n}^{n} F\right\}=\int_{{ }_{n} l}\left[{ }^{0} T\right]^{\mathrm{T}} \cdot\left[{ }^{u} T\right]^{\mathrm{T}} \cdot\left[{ }_{n}^{n} \bar{B}_{L}(0)\right]^{\mathrm{T}} \cdot\left\{{ }_{n}^{n} \bar{R}(0)\right\} \mathrm{d} l$

where the value in parenthesis in $\left[{ }_{n}^{n} \bar{B}_{L}\right]$ indicates the location of the integration point, and that in $\left\{{ }_{n}^{n} \bar{R}\right\}$ indicates the point at which stresses are evaluated.

Using elementary beam theory, relations between bending moments $\bar{R}_{1}, \bar{R}_{2}$ and shear forces $\bar{R}_{5}, \bar{R}_{6}$ can be expressed as

$\bar{R}_{5}=-\frac{\mathrm{d} \bar{R}_{2}}{\mathrm{~d} z}$

$\bar{R}_{6}=-\frac{\mathrm{d} \bar{R}_{1}}{\mathrm{~d} z}$

Thus, the distributions of bending moment increments $\Delta_{n} \bar{R}_{1}(s)$ and $\Delta_{n} \bar{R}_{2}(s)$ along the element length can be approximated by the following equations, using the bending moment increments $\Delta_{n} \bar{R}_{1}(0), \Delta_{n} \bar{R}_{2}(0)$ and the shear force increments $\Delta_{n} \bar{R}_{5}(0), \Delta_{n} \bar{R}_{6}(0)$ at the midpoint of the element:

$\Delta_{n} \bar{R}_{1}(s)=\Delta_{n} \bar{R}_{1}(0)-\frac{\Delta_{n} \bar{R}_{6}(0)^{n} l s}{2}$

$\Delta_{n} \bar{R}_{2}(s)=\Delta_{n} \bar{R}_{2}(0)-\frac{\Delta_{n} \bar{R}_{5}(0)^{n} l s}{2}$

where ${ }^{n} l$ is element length at $t=t_{n}$. Eqs. (37a) and (37b) show that bending moments are subject to a linear change in an element and are likely to take the maximum value on either ends $(s= \pm 1)$. As other resultant forces have constant values in the element, a fully plastic state can be determined with the yield function (14) by comparing the calculated distributions from Eqs. (37a) and (37b).

In dynamic collapse analyses using the ASI technique, an explosion or a fracture is expressed by shifting the numerical integration point according to Eq. (34), immediately after the occurrence of a fractured section on either end of the element and reducing the resultant forces of the element simultaneously. For instance, if a fully plastic section or a fractured section has first occurred at the left end of an element $(r=-1)$, the numerical integration point is shifted im- 
mediately to the right end of the element $(s=1)$ according to Eq. (34). The released force vector, which operates on the element at the next step in the analysis, is then expressed by the following equation:

$$
\left\{{ }_{n}^{n} F\right\}=\int_{{ }_{n} l}\left[{ }^{0} T\right]^{\mathrm{T}} \cdot\left[{ }^{u} T\right]^{\mathrm{T}} \cdot\left[{ }_{n}^{n} \bar{B}_{L}(1)\right]^{\mathrm{T}} \cdot\left\{{ }_{n}^{n} \bar{R}(-1)\right\} \mathrm{d} l
$$

Similarly, if a fully plastic section or a fractured section has first occurred at the right end of the element $(r=1)$, the numerical integration point is shifted to the left end of the element $(s=-1)$.

In case of the implicit scheme the incremental stiffness matrices and initial stress matrices used in the algorithm, for the case when the entire region in an element is elastic, are

$$
\begin{aligned}
& {\left[{ }_{n}^{n} \bar{K}_{L}\right]=\int_{{ }_{n} l}\left[{ }^{u} T\right]^{\mathrm{T}} \cdot\left[{ }^{0} T\right]^{\mathrm{T}}\left[{ }_{n}^{n} \bar{B}_{L}(0)\right]^{\mathrm{T}}\left[D^{e}(0)\right]\left[{ }_{n}^{n} \bar{B}_{L}(0)\right]} \\
& \times\left[{ }^{0} T\right] \cdot\left[{ }^{u} T\right] \mathrm{d} l \\
& {\left[{ }_{n}^{n} \bar{K}_{N L}\right]=\int_{{ }_{n}}\left[{ }^{u} T\right]^{\mathrm{T}} \cdot\left[{ }^{0} T\right]^{\mathrm{T}}\left[{ }_{n}^{n} \bar{G}(0)\right]^{\mathrm{T}}\left[{ }_{n}^{n} \bar{S}(0)\right]\left[{ }_{n}^{n} \bar{G}(0)\right]} \\
& \times\left[{ }^{0} T\right] \cdot\left[{ }^{u} T\right] \mathrm{d} l
\end{aligned}
$$

In case the section is fully plastic or has fractured first at the left end of the element $(r=-1)$, the incremental stiffness matrices and initial stress matrices are given by the following equation:

$$
\begin{aligned}
{\left[{ }_{n}^{n} \bar{K}_{L}\right]=} & \int_{{ }^{n} l}\left[{ }^{u} T\right]^{\mathrm{T}} \cdot\left[{ }^{0} T\right]^{\mathrm{T}}\left[{ }_{n}^{n} \bar{B}_{L}(1)\right]^{\mathrm{T}}\left[D^{p}(-1)\right] \\
& \times\left[{ }_{n}^{n} \bar{B}_{L}(1)\right]\left[{ }^{0} T\right] \cdot\left[{ }^{u} T\right] \mathrm{d} l \\
{\left[{ }_{n}^{n} \bar{K}_{N L}\right]=} & \int_{{ }^{n} l}\left[{ }^{u} T\right]^{\mathrm{T}} \cdot\left[{ }^{0} T\right]^{\mathrm{T}}\left[{ }_{n}^{n} \bar{G}(1)\right]^{\mathrm{T}}\left[{ }_{n}^{n} \bar{S}(-1)\right] \\
& \times\left[{ }_{n}^{n} \bar{G}(1)\right]\left[{ }^{0} T\right] \cdot\left[{ }^{u} T\right] \mathrm{d} l
\end{aligned}
$$

It should be noted that when new hinges are formed, the resultant force increments calculated at the new integration point are automatically added to those originally existing at the very point. As a result of using this procedure, a 'non-smoothness' type of phenomenon does not appear in the calculation. More details of the implicit ASI algorithm are explained in the authors' previous paper [9].

The conceptualization of explosion or member fracture in the ASI technique is shown in Fig. 2, by indicating the location of the numerical integration point in the linear Timoshenko beam element along with the rotational and shear springs in the RBSM. When a

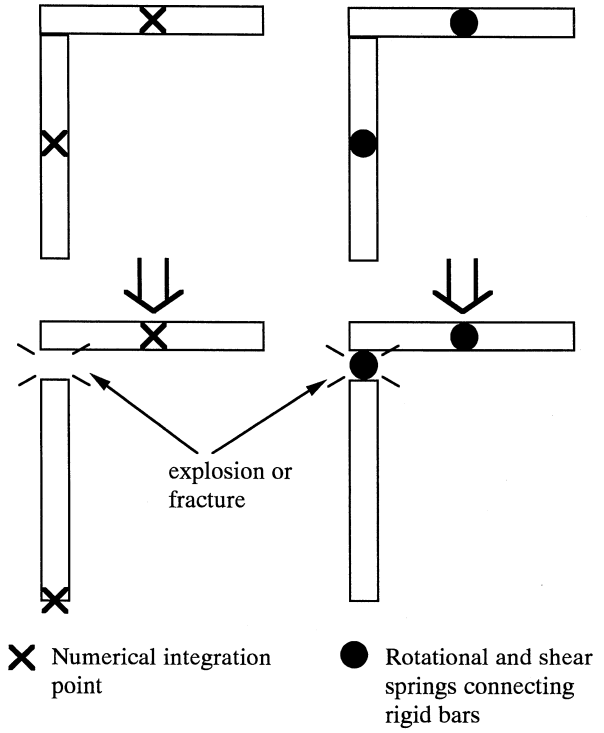

Fig. 2. Conceptualization of explosion or member fracture in ASI technique.

member fracture occurs in an element, the numerical integration point is shifted to the opposite end of the fractured section from the center of the element.

The release of resultant forces acting on the section might produce longitudinal waves in the element, when the member fractures. Therefore, a gradual release process or artificial viscosity is generally introduced to reduce this effect, for stabilizing the numerical calculation. However, in this paper, an instant release technique is used to preserve the simplicity of the algorithm. Likewise, rebounds and insertions into the ground or contact between members are neglected in this algorithm.

It is also to be noted that new imaginary nodal points, indicating fractured sections for a post processor, are required to be introduced after the occurrence of member fracture, even though the elements and nodal points are linked in the numerical model. The elements with imaginary nodal points are then visualized as rigid bars thereafter.

\section{Dynamic collapse analyses of reinforced concrete building frames}

In this section, the explicit nonlinear code based on the ULF adaptation of the ASI technique is implemented into an existing finite element code and is then applied to an explosive demolition analysis of a five storied, five span reinforced concrete building 


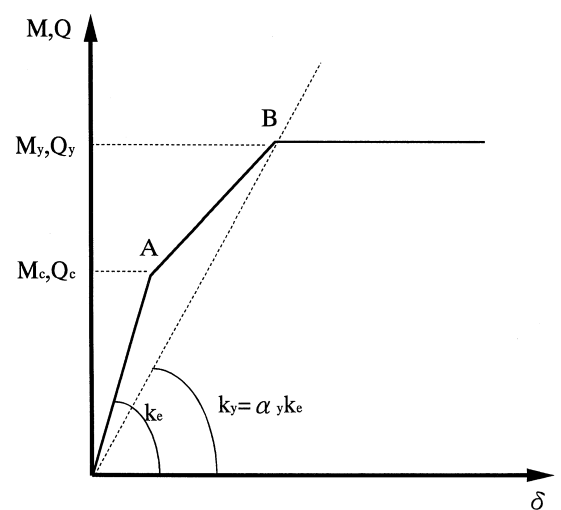

Fig. 3. Tri-linear model for reinforced concrete.

frame. Secondly, a seismic damage analysis is carried out on the same model by an implicit nonlinear code based on the ULF adaptation of the ASI technique.

\subsection{Strength characteristics of the $R C$ member used in the analysis}

The strength characteristics of the RC member used in the analyses is discussed in this section. Generally, many parameters such as shape, steel bar arrangement, material characteristics as well as stress condition or stress history have big influences on the strength characteristics of RC members. Consequently, there are limits to the application ranges of proposal formulas and in this paper, formulas which does not require strict limitations, are used [15].

A tri-linear type model, as shown in the force-displacement relation curve in Fig. 3, which has a crack point $\mathrm{A}$ and an yield point $\mathrm{B}$, is employed. The formulas of crack and yield strengths are as follows:

- Flexural crack strength.

$$
M_{\mathrm{c}}=1.8 \sqrt{F_{\mathrm{c}}} \cdot Z_{\mathrm{e}}+\frac{N Z_{\mathrm{e}}}{A_{\mathrm{c}}}
$$

- Flexural yield strength.

$$
M_{\mathrm{y}}=0.8 a_{\mathrm{t}} \cdot f_{\mathrm{s}} \cdot D+0.5 N D\left(1-\frac{N}{b D F_{\mathrm{c}}}\right)
$$

- Shear crack strength.

$$
Q_{\mathrm{c}}=\left(1+\frac{N}{150 b D}\right) k_{\mathrm{c}}\left(500+F_{\mathrm{c}}\right) \frac{0.085}{M / Q d+1.7} b j
$$

- Shear ultimate strength.

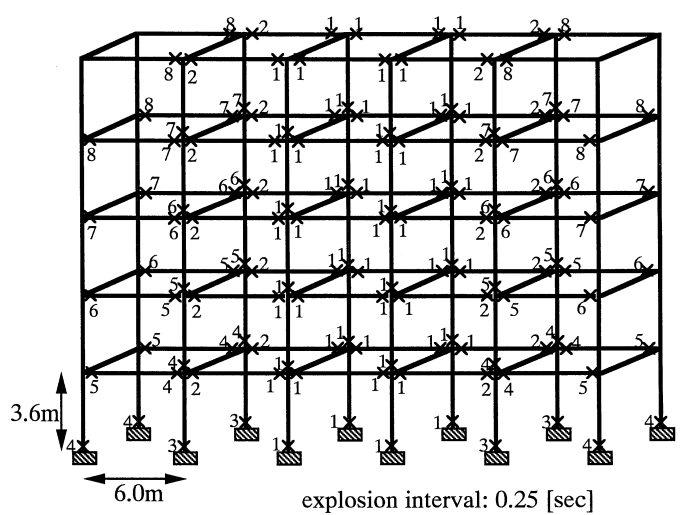

Fig. 4. Five storied five span RC building frame model used in explosive demolition analysis.

$$
\begin{aligned}
Q_{\mathrm{y}}= & {\left[\frac{0.115 k_{\mathrm{u}} \cdot k_{\mathrm{p}}\left(180+F_{\mathrm{c}}\right)}{M / Q d+0.115}+2.7 \sqrt{p_{w} \cdot \mathrm{s} f_{\mathrm{wy}}}\right.} \\
& \left.+0.1 \frac{N}{b D}\right] b j
\end{aligned}
$$

- Stiffness decrease ratio at yield point.

$$
\begin{aligned}
\alpha_{\mathrm{y}}= & \left(0.043+1.65 n \cdot p_{\mathrm{t}}+0.043 \frac{M}{Q d}+0.33 \eta_{0}\right) \\
& \times\left(\frac{d}{D}\right)^{2}
\end{aligned}
$$

where $b$ is column width, $D$ the column height, $h_{0}$ the inner measured height of the column, $d$ the equivalent height $=0.9 D, A_{\mathrm{c}}$ the cross-sectional area of the RC member $=b D, F_{\mathrm{c}}$ the compressive strength of concrete, $f_{\mathrm{y}}$ the yield stress of tension reinforcement, $f_{\mathrm{wy}}$ the tension yield stress of shear reinforcement, $Z_{\mathrm{e}}$ the section modulus considering reinforcement $=1.1 Z=1.1 \frac{b D^{2}}{6}, n$ the ratio of Young's moduli of concrete and reinforcement, $N$ the normal force, $M / Q d$ the shear span ratio $=\frac{h_{0}}{2 d}, \eta_{0}$ the normal force ratio $=\frac{N}{A_{\mathrm{c}} F_{\mathrm{c}}}, j$ the distance between the center of stresses $=\frac{7}{8} d, k_{\mathrm{c}}$ the correction factor of the member height $=0.7, k_{\mathrm{u}}$ the correction factor of member dimension $=0.7, k_{\mathrm{p}}$ the correction factor of tension reinforcement ratio $=0.82 p_{\mathrm{t}}^{0.23}, p_{\mathrm{t}}$ the tension reinforcement ratio $=\frac{a_{\mathrm{t}}}{b D}, p_{\mathrm{w}}$ the shear reinforcement ratio $=\frac{a_{\mathrm{t}}}{b \gamma}, a_{\mathrm{t}}$ the cross-sectional area of tension reinforcement, $a_{\mathrm{w}}$ the cross-sectional area of a pair of shear reinforcement and $\chi$ the distance between each shear reinforcement.

\subsection{Explosive demolition analysis of an $R C$ building frame}

The proposed explicit nonlinear code based on the ULF adaptation of the ASI technique is employed in 

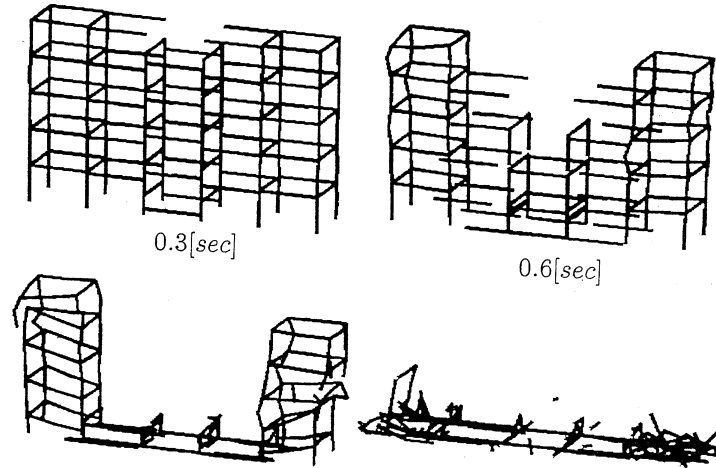

$1.0[\mathrm{sec}]$

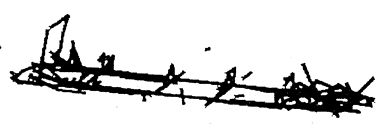

$3.0[\mathrm{sec}]$

Fig. 5. Explosive demolition analysis of RC building frame using ASI technique.

an explosive demolition analysis of a five storied, five span RC building frame shown in Fig. 4. The members of the frame are divided into two Timoshenko beam elements. Dead loads are assigned statically to the nodal points at the initial step of the analysis, followed by dynamic sequenced explosions of member sections in order and time as shown in the figure.

The material properties used in the analysis are as follows: Young's modulus of concrete $E_{\mathrm{c}}=2.1 \times 10^{5}$ $\mathrm{kg} / \mathrm{cm}^{2}$, Poisson's ratio of concrete $v_{\mathrm{c}}=0.17$, density of RC member $\rho=2.4 \times 10^{-3} \mathrm{~kg} / \mathrm{cm}^{3}$, compressive strength of concrete $F_{\mathrm{c}}=240 \mathrm{~kg} / \mathrm{cm}^{2}$, yield stress of tension reinforcement ${ }_{s} f_{y}=4.0 \times 10^{3} \mathrm{~kg} / \mathrm{cm}^{2}$, and tension yield stress of shear reinforcement ${ }_{\mathrm{s}} f_{\mathrm{wy}}=3.0 \times 10^{3}$ $\mathrm{kg} / \mathrm{cm}^{2}$. Member fracture is assumed to occur at the critical curvature of $\kappa_{\mathrm{cr}}=1.0 \times 10^{-3} 1 / \mathrm{mm}$. The columns on the first floor are $80 \times 80 \mathrm{~cm}$, and those on the second and higher floors are $60 \times 60 \mathrm{~cm}$. All beams have a section of $40 \times 40 \mathrm{~cm}$. The main reinforcement ratio is $4 \%$ for columns below the third floor, $0.8 \%$ (minimum allowable ratio) for columns on upper floors, and $5 \%$ for lateral beams. The diameters of the shear reinforcement in all members are set to $1.3 \mathrm{~cm}$, and the spacing between shear reinforcements $\chi$ is $30 \mathrm{~cm}$.

The theoretical maximum time increment $\Delta t_{\mathrm{cr}}$ for this model, according to Courant's stability condition Eq. (25), is $\Delta t_{\mathrm{cr}}=6.15 \times 10^{-4} \mathrm{~s}$. The time increment is selected as $\Delta t=5.00 \times 10^{-4} \mathrm{~s}$ to avoid any instabilities. The total number of time steps used in the analysis is 10,000 and the computing time taken using an EWS (Sun SPARC station 5) is about $110 \mathrm{~min}$.

One of the requirements for successful demolition is that floor slabs should fragment into small pieces. Therefore, the explosions are positively sequenced to occur outward starting from the central core as shown

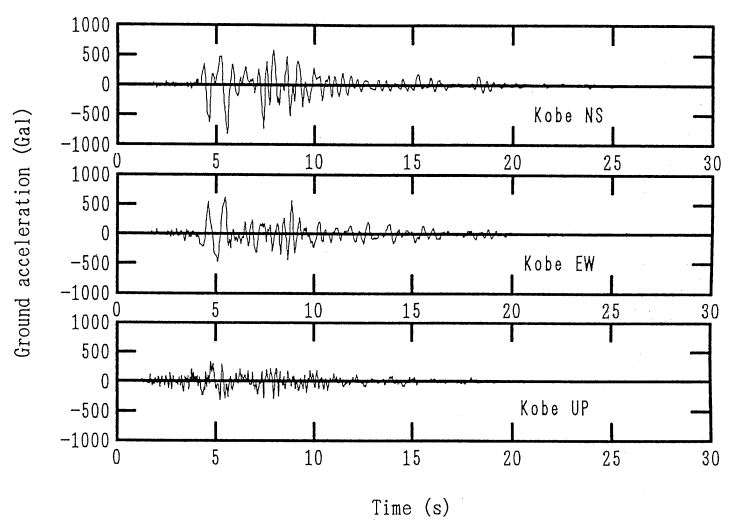

Fig. 6. Great Hanshin-Awaji Earthquake accelerogram used in seismic damage analysis.

in Fig. 4, thus causing a destructive shearing distortion between the stories. As shown in the deformed configurations of the $\mathrm{RC}$ building frame in Fig. 5 the whole structure collapses inward as expected, as explosions occur one after another at several beam sections. Since contact between members is neglected in the model, phenomena different from those observed in actual demolitions such as beams going through floors, appear in the configurations.

Nevertheless, it is seen that the numerical scheme is able to model the most important features of an explosive demolition process. The computational efficiency and the practicability of using the proposed explicit nonlinear code are also confirmed.

\subsection{Seismic damage analysis of an RC building frame}

In this section, the proposed implicit nonlinear code based on the ULF adaptation of the ASI technique, is employed in a seismic damage analysis of the $\mathrm{RC}$ building frame used in the previous section. As in the explosive demolition analysis, dead loads are applied statically to the nodal points at the initial step of the analysis. A dynamic analysis of the frame is performed with the fixed points of the model excited by threedirectional seismic wave (Great Hanshin-Awaji Earthquake, 1995, JMA Kobe), as shown in Fig. 6.

Newmark's $\beta$ method $(\delta=1 / 2)$, well known for its practicability and usefulness, is chosen for the direct numerical integration scheme in this analysis. However, problems tend to arise, when there are strong nonlinearities, if the value of $\beta$ is selected in the range of $0<\beta \leq 1 / 4$. On the other hand, the results tend to converge in a stable manner, if the range of $1 / 4<\beta<$ $1 / 2$ is selected [16] although there are slight limitations to accuracy. Therefore, a value of $\beta=0.4$ is selected in 


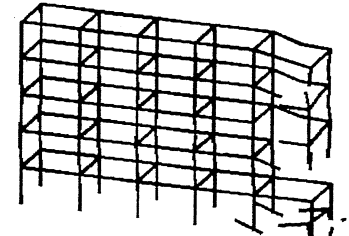

$10.8[\mathrm{sec}]$

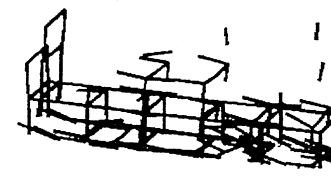

$13.8[\mathrm{sec}]$

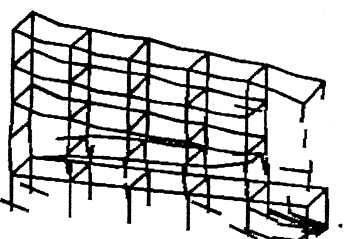

$12.6[\mathrm{sec}]$

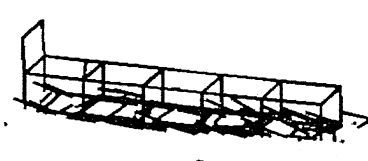

$8.0[\mathrm{sec}]$
Fig. 7. Seismic damage analysis of RC building frame using ASI technique.

the analysis. The time increment used in this analysis is $\Delta t=7.50 \times 10^{-3} \mathrm{~s}$, and the input time of the seismic wave is $30 \mathrm{~s}$ (4,000 steps). The computing time taken by the EWS (Sun SPARC station 5) is about $190 \mathrm{~min}$.

Fig. 7 shows the collapse sequence of the RC building frame. Fracture occurs first at a column member on the second floor, which has lost its strength by repeated yielding and unloading, followed by a collapse chain reaction of columns and beams supporting the third floor and above. The collapse seen in columns on the upper floor is in a way inevitable, because the main reinforcement ratio in the columns on the upper floor is reduced to the minimum allowable ratio, which is actually a commonly used practice in Japan.

The results of the analysis confirm that the proposed numerical scheme is able to model seismic damages observed in actual eqrthquakes. In addition, the algorithm is seen to be economical and practical even though an implicit nonlinear code is used.

\section{Concluding remarks}

In the present paper, a nonlinear finite element code using the ASI technique, is extended and applied to problems of structural discontinuities. Fracture of a section is modeled by shifting the numerical integration point with a simultaneous release of the resultant forces. The algorithm is implemented into an existing FEM code, and several numerical tests are carried out. The results show that this technique can be used in numerical estimation of structural reliabilities. However, the algorithm needs to be improved by considering contact between members so as to obtain results that agree better with actual behavior.

\section{Acknowledgements}

This work was partially supported by the University of Tsukuba Research Projects Grant.

\section{References}

[1] Cundall PA. A computer model for simulating progressive, largescale movement in blocky rock system. Proceedings of the International Symposium on Rock Mechanics 1971;II-8:129-36.

[2] Shi GH, Goodman RE. Discontinuous deformation analysis. Proceedings of 25th U.S. Symposium on Rock Mechanics 1984;1:269-77

[3] Meguro K, Hakuno M. Simulation of collapse process of structures due to earthquake (in Japanese). Proceedings of Symposium on Computational Methods in Structural Engineering and Related Fields 1991;15:325-30.

[4] Itoh M, Yoshida N, Utagawa N, Kondo I, Simulation of blast demolition of reinforced concrete buildings. Proceedings of the Third World Congress on Computational Mechanics 1994;2:1152-53.

[5] Ma MY, Barbeau P, Penumadu D. Evaluation of active thrust on retaining walls using DDA. Journal of Computing in Civil Engineering 1995;1:820-7.

[6] Kondo I, Utagawa N, Ito M, Yoshida N. Numerical method to simulate collapse behavior in blasting demolition of space framed structure (in Japanese). Proceedings of the 13th Symposium on Computer Technology of Information, Systems and Applications 1990;1:49-54.

[7] Yarimer E. Demolition by controlled explosion as a dynamical process. Structures under Shock and Impact 1989, pp. 411-16.

[8] Tosaka N, Kasai Y, Honma T. Computer simulation for felling patterns of building. Demolition Methods and Practice 1988, pp. 395-403.

[9] Toi Y, Isobe D. Adaptively Shifted Integration technique for finite element collapse analysis of framed structures. International Journal for Numerical Methods in Engineering 1993;36:2323-39.

[10] Toi Y, Isobe D, Adaptively Shifted Integration technique for nonlinear finite element analysis of framed structures. Proceedings of the Third World Congress on Computational Mechanics 1994;2:1682-83.

[11] Toi Y, Isobe D. Adaptively Shifted Integration technique for nonlinear finite element analysis of large-scale framed structures. Bulletin of Earthquake Resistant Structure Research Center 1994;27:13-9.

[12] Toi Y, Isobe D. Finite element analysis of quasi-static and dynamic collapse behaviors of framed structures by the adaptively shifted integration technique. Computers and Structures 1996;58:947-55.

[13] Toi Y. Shifted Integration technique in one-dimensional 
plastic collapse analysis using linear and cubic finite elements. International Journal for Numerical Methods in Engineering 1991;31:1537-52.

[14] Bathe KJ. In: Finite element procedures. Englewood Cliffs, NJ: Prentice Hall, 1995, pp. 565-6.
[15] Architectural Institute of Japan, Guidelines on ultimate strength design of reinforced concrete (in Japanese), 1988, pp. 70-71.

[16] Shimizu N, Response analysis using personal computers (in Japanese), Kyouritu-Shuppan 1989, pp. 267-68. 